\title{
Effects of Air Pollutant Exposure on Exacerbation Severity in Asthma Patients with or without Reversible Airflow Obstruction
}

\author{
Wei Fangl,* \\ Yu Zhang ${ }^{1} * *$ \\ Sinian $\mathrm{Li}^{1, *}$ \\ Aiming $\operatorname{Liu}^{2, *}$ \\ Yin Jiang ${ }^{2}$ \\ Dandan Chen' \\ Binbin $\mathrm{Li}^{3}$ \\ Can $\mathrm{YaO}^{3}$ \\ Rongchang Chen' \\ Fei Shi ${ }^{3}$
}

'Key Laboratory of Shenzhen Respiratory Diseases, Institute of Shenzhen

Respiratory Diseases, Shenzhen People's Hospital (The First Affiliated Hospital of Southern University of Science and Technology, The Second Clinical Medical College of Jinan University), Shenzhen, Guangdong, People's Republic of China;

${ }^{2}$ Shenzhen National Climate

Observatory, Meteorological Bureau of Shenzhen Municipality, Shenzhen Key Laboratory of Severe Weather in South China, Shenzhen, Guangdong, People's Republic of China; ${ }^{3}$ Emergency

Department, Institute of Shenzhen Respiratory Diseases, Shenzhen People's Hospital (The First Affiliated Hospital of Southern University of Science and Technology, The Second Clinical Medical College of Jinan University), Shenzhen, Guangdong, People's Republic of China

*These authors contributed equally to this work
Background: Reversible airflow obstruction (RO) and fixed airflow obstruction (FO) are two important clinical phenotypes of asthma. However, the relationship between air pollutant exposure and exacerbation of the two phenotypes is unknown.

Objective: To study the effects of air pollutant exposure on exacerbation severity in asthma patients with or without FO.

Methods: A total of 197 severe asthma patients were enrolled, and divided into two groups: the FO group $(n=81)$ and the RO group $(n=116)$. We collected the demographic data, laboratory parameters, pulmonary function test parameters, and the daily average concentrations of different air particles in Shenzhen on the different lag days of each subject. The receiver operating characteristic (ROC) curve was used to identify the effects of major air pollutants on the severity of asthma patients with RO.

Results: Compared with the RO group, the FO group had fewer women, lower body mass index (BMI), longer disease duration, higher smoking history rate, allergic family history rate, FeNO level, and lower levels of large airway parameters. The median exposure levels of PM10 and PM2.5 in the severe RO subgroup were both higher than those in the mild-tomoderate RO subgroup on Lag0, 1 and 3, and the median exposure level of PM1 on Lag0 in the severe RO subgroup was significantly higher than that in the mild-to-moderate RO subgroup. Logistic regression modeling indicated exposure to PM2.5 and PM1.0 on Lag0, and PM10 on Lag0-2 were the independent risk factors for hospital admissions for asthma patients with RO. By performing an ROC curve analysis, PM2.5 on $\operatorname{Lag} 0$ (AUC $=0.645, p=$ 0.027 ) provided a best performance to predict severe asthma exacerbations with RO, with a sensitivity of $36.0 \%$ and a specificity of $91.2 \%$.

Conclusion: Short-term exposure to PM10, PM2.5 and PM1 may play a role in exacerbation severity among asthma patients with RO.

Keywords: air particles, asthma, reversible airflow obstruction, fixed airflow obstruction

\section{Introduction}

Asthma is a common chronic respiratory disease characterized by airway inflammation, hyper-responsiveness of the respiratory tract and reversible airway obstruction (RO). ${ }^{1}$ Among the world population, about one-third are suffering from asthma, and there are annual deaths of almost 2.5 million asthma patients because of severe exacerbation. In recent years, the prevalence of asthma has been increasing, resulting in substantial economic costs of patients due to frequent hospitalization. ${ }^{2}$
Correspondence: Rongchang Chen; Fei Shi Email chenrc@vip.163.com; shi.fei@szhospital.com 
Airway remodeling is a major pathological change, and can be observed from early onset of asthma, leading to fixed airflow obstruction (FO) in some asthma patients. ${ }^{3}$ The phenotype of FO (as defined by forced expiratory volume in 1 second [FEV1)/forced vital capacity (FVC] $\leq 0.7$ at baseline after appropriate asthma treatment for 6 months) is associated with greater disease burden and higher frequency of asthma exacerbations. ${ }^{4,5}$ The underlying mechanisms of FO phenotype are poorly understood, while the research evidence suggests that airway remodeling, increased lung compliance and air-trapping may contribute to FO. ${ }^{6}$ Therefore, airflow limitation may be partially reversible in asthma with FO. Indeed, patients with severe and difficult-to-treat asthma may be associated with an accelerated decline in lung function that causes FO. These patients have fewer sensitivities to asthma medication, and may find it difficult to recover from an asthma attack even after taking regular medication. ${ }^{7}$

It has been confirmed that asthma is likely to result from an interaction between genetic and environmental factors. ${ }^{8}$ Aside from some common allergens including pollen, dust mites and perfume, air pollution exposure also plays an important role for severe exacerbations that result in increased in-hospital and short-term mortality. ${ }^{9}$ At present, it has been found that inhaling certain particles in the atmosphere may be enough to induce acute asthma attacks, and exacerbate small airways injury and dysfunction among some patients with asthma. ${ }^{10}$ However, the potential adverse health effects of exposure to airborne particles on asthma patients with different clinical phenotypes is not well known.

Shenzhen, located in the south of China, is one of the most developed Chinese cities, with a population exceeding 20 million. According to the reports of Shenzhen government, ambient concentrations of PM2.5 and PM10 are out of bounds of the World Health Organization standard. Moreover, exposure to some environmental factors can trigger and exacerbate asthma. Therefore, we aimed to study the relationship between the exposure to airborne particles and asthma patients with or without FO in Shenzhen, and target asthma prevention more precisely through environmental intervention strategies.

\section{Methods}

\section{Study Subjects}

This study was retrospectively carried out with 197 subjects with acute exacerbation of asthma (Figure 1) admitted to Pulmonary Department, Shenzhen People's Hospital (the First Affiliated Hospital of Southern University of Science and Technology, the Second Clinical Medical College of Jinan University), from January 2018 to August 2019. The medical records were consecutively collected and reviewed. Inclusion criteria were physician-diagnosed asthma according to 2017 Global Initiative for Asthma (GINA)

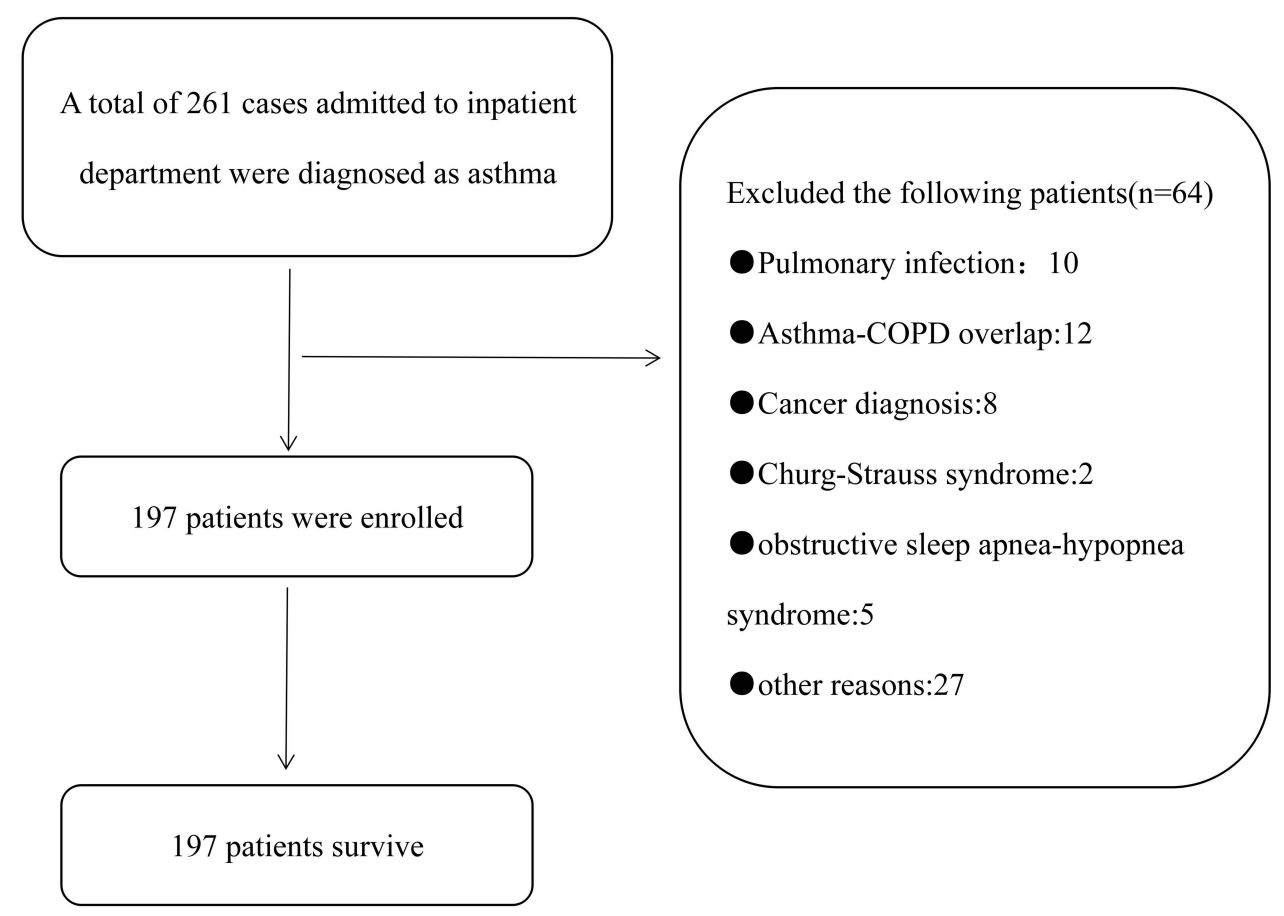

Figure I Screening for and enrollment in the study. 
guidelines. ${ }^{11}$ Subjects were excluded if they had a history of autoimmune or other lung diseases, including but not limited to COPD, asthma-COPD overlap, pneumonia, bronchiectasis, obstructive sleep apnea-hypopnea syndrome, cystic fibrosis, interstitial lung disease, and lung cancer. In addition, patients with unavailable PM2.5 data were also excluded. The included subjects were classified into two major groups: those with FO (post-bronchodilator [BD] and on-treatment ratio of FEV1/FVC persistently below 0.7 ), and those with RO (post-BD and/or on-treatment ratio of FEV1/FVC greater than 0.7). ${ }^{12,13}$ Both groups were then divided into two subgroups based on their illness severity (mild-to-moderate or severe) according to GINA2017: (1) the mild-to-moderate fixed limitation group included 42 subjects with FO due to mild-to-moderate asthma; (2) the severe fixed limitation group included 39 subjects with FO due to severe asthma; (3) the mild-tomoderate reversible obstruction group included 91 subjects with RO due to mild-to-moderate asthma; and (4) the severe reversible limitation group included 25 subjects with RO due to severe asthma. The clinical retrospective data were retrieved from the medical records of all subjects, including demographic characteristics, clinical features, pulmonary function tests (PFTs), laboratory results, radiological findings, therapies and 28-day clinical outcomes. Two physicians (S.L. and Y.Z.) independently reviewed the data. The time course of the main air pollutants in Shenzhen from September 2017 to August 2019 is shown in Figure 2.

The study was conducted according to the principles of the Declaration of Helsinki, and approved by the Ethics Committee of Shenzhen People's Hospital (LL-KT -20151230). No analyzed data contained personally identifiable information and therefore no informed consent was required.

\section{PFT Measurements}

Post-BD and on-treatment PFT were performed using a V6200 Series Autobox (Sensor Medics, USA) spirometer device following ATS/ERS standards. ${ }^{14}$ PFT parameters like FEV1, FEV1 as a percentage of predicted FVC (FEV1\%pred), FVC as a percentage of predicted FVC (FVC\%pred), and FEV1/FVC ratio were collected. A predicted ratio for each parameter was calculated based on age, sex, height, and race.

\section{Measurement of Ambient PM2.5 Concentrations}

Data on ambient particulate matter (PM) 2.5 and other air pollutants (including PM10, PM1.0, sulfur dioxide $\left[\mathrm{SO}_{2}\right]$, nitrogen dioxide $\left[\mathrm{NO}_{2}\right]$, ozone $\left[\mathrm{O}_{3}\right]$, and carbon monoxide [CO]), temperature, and humidity on the different lag days of each subject were obtained from Shenzhen National

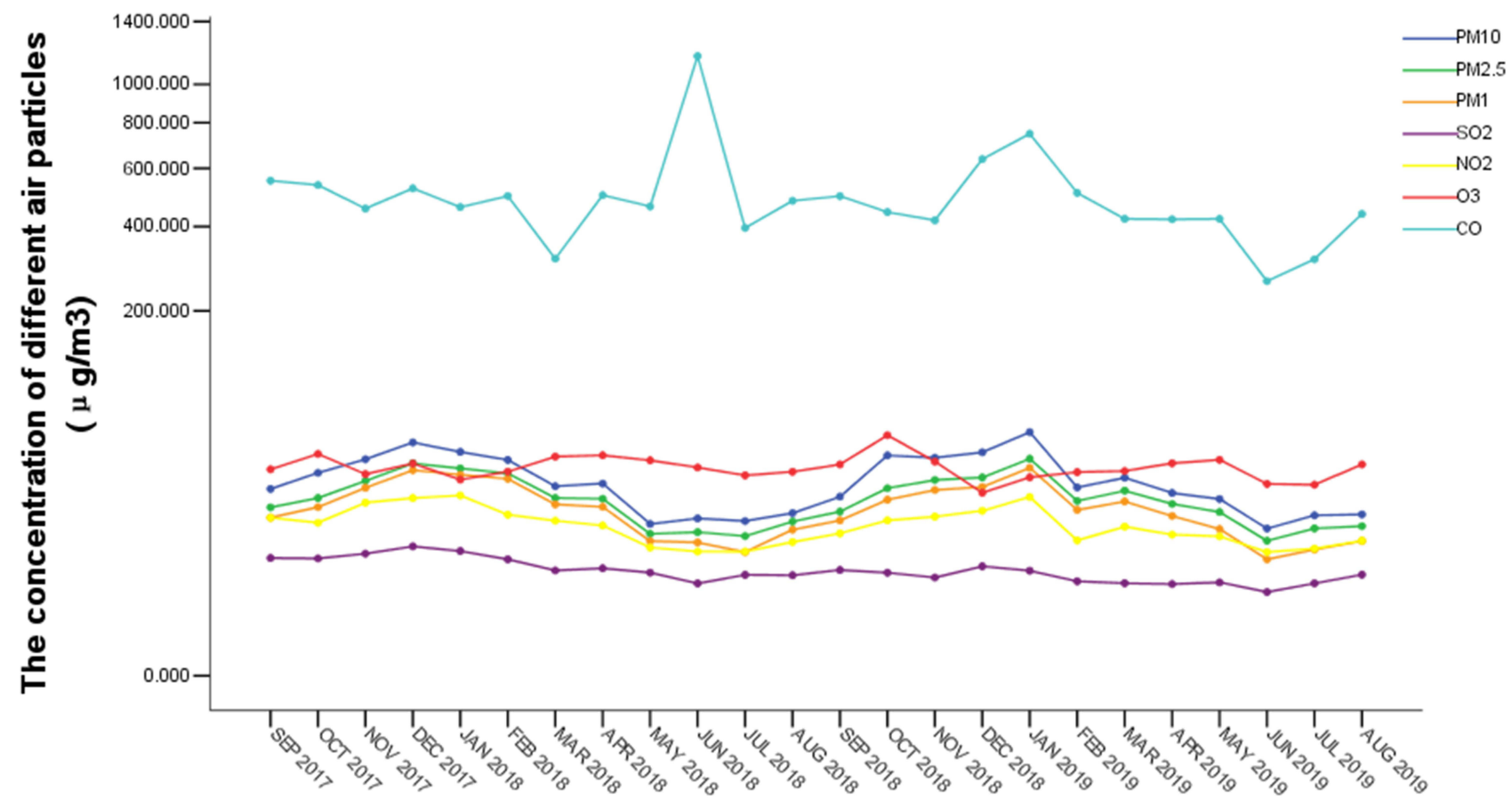

Figure 2 The time course of main air pollutants in Shenzhen from September 2017 to August 2019. 
Climate Observatory. We used a single-day lag (from Lag0 to Lag3) model to explore the short-term effects of air pollutant exposure. A 0-3-day lag refers to asthma onset due to air pollutant exposure on the same day (Lag0), 1 day before ( $\operatorname{Lag} 1), 2$ days before ( $\operatorname{Lag} 2)$, and 3 days before (Lag3). The 24-hour mean concentrations of the ambient air pollutants were analyzed using real-time monitoring data within Shenzhen.

\section{Statistical Evaluation}

SPSS 13.0 software (SPSS Inc., Chicago, IL, USA) was used for statistical evaluations. Parametric and non-parametric data are presented as means (standard deviation, SD) and medians (quartile 1-quartile 3). For comparison of 4 groups for parametric and non-parametric variables, the one-way analysis of variance (ANOVA) or Kruskal-Wallis test was used and the $\chi^{2}$ test was used for proportions. Linear correlation regression was used to certify whether there was a correlation between each air pollutant's concentrations and the severity of disease. Receiver operating characteristic (ROC) curves were generated, and the area under the curve (AUC) was calculated to identify suitable parameters for distinguishing between two groups. A $p$-value of less than $0.05(p<0.05)$ was considered a statistically significant difference.

\section{Results}

\section{Baseline Characteristics of All Subjects}

We enrolled a total of 197 asthma patients (82 men and 115 women) aged 23-73 years in this study (Figure 1). The obtained medical data and demographic characteristics of the four study populations are presented in Table 1. The asthma patients with FO comprised fewer women (46.91 vs $66.38 \% ; p=0.006$ ), lower body mass index (BMI; 23.19 \pm 3.12 vs $\left.24.41 \pm 3.25 \mathrm{~kg} / \mathrm{m}^{2} ; p=0.009\right)$, longer disease duration (60 $[12.0,240.0]$ vs $36[5.0,96.0]$ month; $p=0.002])$, and higher smoking history rate $(35.80$ vs $15.52 \% ; p=0.002)$ than those with RO. It is interesting to note that the family history of allergy and FeNO levels were significantly higher in the group with FO in comparison to the group with RO (family history of allergy: 25.93 vs $6.03 \%$, FeNO: 35.00 [16.00, $64.25]$ vs 20.50 [12.00, 43.50]; $p<0.001$ and $p=0.006$, respectively]). However, there was no statistical difference in ages, length of hospital stay, and pack-years of smoking between the two groups ( $p=0.554,0.475$, and 0.191 , respectively). Moreover, the PFT parameters including FEV1 (1.85 \pm 0.74 vs $2.60 \pm 0.74 \mathrm{~L} ; p<0.001)$, FEV1\%pred (63.54 \pm 17.78 vs $95.06 \pm 14.59 \% ; p<0.001)$, FVC $(3.08 \pm 1.00$ vs $3.38 \pm 0.90$; $p=0.029), \quad \mathrm{FVC} \%$ pred $(87.72 \pm 17.48$ vs $103.75 \pm 15.87 \%$; $p<0.001)$, and FEV1/FVC ratio $(58.41 \pm 8.54$ vs $76.45 \pm 4.52$; $p<0.001$ ) of the FO group were all lower than those of the RO group. There was no difference between the two groups in the use of short-term inhaled budesonide and systemic corticosteroids ( $p=0.141$ and 0.940 , respectively). The details of the clinical data in the four subgroups are shown in Table 1.

\section{Different Levels of Air Pollutant Exposure Between the Subgroups}

We compared the results of ambient air monitoring within the four subgroups (Table 2). Suppose that lag indicates exposure-time period defined by time since exposure. There was a statistically significant difference between the two RO subgroups in terms of PM10, PM2.5, PM1 and NO2 on different lag days. The median exposure levels of PM10 and PM2.5 in the severe RO subgroup were both higher than those in the mild-to -moderate RO subgroup on the day of asthma onset (Lag0) (PM10: 32.76 vs 20.70, PM2.5: 23.15 vs 16.08; $p=0.031$ and 0.027 , respectively), one day before asthma onset (Lag1) (PM10: 34.89 vs 19.47, PM2.5: 24.96 vs 16.24; $p=0.032$ and 0.033 , respectively) and three days before asthma onset (Lag3) (PM10: 32.72 vs 19.67, PM2.5: 22.71 vs.15.69; $p=0.008$ and 0.023 , respectively). Meanwhile, the median exposure level of PM1 on Lag0 in the severe RO subgroup was significantly higher than that in the mild-to-moderate RO subgroup (18.09 vs 12.71; $p=0.043$ ). Furthermore, exposure levels of $\mathrm{NO}_{2}$ on Lag3 showed a higher median level in the severe RO subgroup than in the mild-to-moderate RO subgroup ( 14.48 vs $7.97 ; p=0.019$ ). Moreover, no significant differences in the levels of main air pollutants (including PM2.5, PM10, PM1.0, $\mathrm{SO}_{2}, \mathrm{NO}_{2}, \mathrm{O}_{3}, \mathrm{CO}$ ) on different lag days were found between the two FO subgroups.

\section{Risk Factors of Hospital Admissions for Asthma Patients with or without RO}

With the univariate and multivariate logistic data analysis, we found that exposure to PM10, PM2.5, PM1.0 on Lag0 were the independent risk factors for hospital admissions for asthma patients with RO (PM10: $\mathrm{OR}=1.057, \quad 95 \% \quad \mathrm{CI} 1.002-1.115 ; \mathrm{PM} 2.5: \quad \mathrm{OR}=1.130$, 95\% CI1.033-1.236; PM1.0: OR=1.150, 95\% CI $1.035-1.277 ; \quad p=0.042,0.007,0.009$, respectively). Moreover, exposure to PM10 on Lag1 (OR=1.127; 
Table I Comparison of Demographic Characteristics Between Fixed Airflow Obstruction Group and Reversible Airflow Obstruction Group

\begin{tabular}{|c|c|c|c|c|c|c|c|}
\hline & \multicolumn{3}{|c|}{ Fixed Airflow Obstruction Group $(n=81)$} & \multicolumn{3}{|c|}{ Reversible Airflow Obstruction Group $(n=\mid 16)$} & \multirow[t]{2}{*}{$p$-value } \\
\hline & Total & $\begin{array}{l}\text { Mild-to- } \\
\text { Moderate }\end{array}$ & Severe & Total & $\begin{array}{l}\text { Mild-to- } \\
\text { Moderate }\end{array}$ & Severe & \\
\hline Age (year), mean $(S D)$ & $49.83(12.32)$ & $46.31(12.17)$ & $53.62(11.47)$ & $48.66(15.15)$ & $47.98(15.16)$ & $51.16(15.19)$ & 0.554 \\
\hline Sex, male, n (\%) & $43(53.09)$ & $24(57.14)$ & $19(48.72)$ & $39(33.62)$ & $32(35.16)$ & $7(28)$ & $0.006 *$ \\
\hline BMI $\left(\mathrm{kg} / \mathrm{m}^{2}\right)$, mean $(\mathrm{SD})$ & $23.19(3.12)$ & $22.99(3.37)$ & $23.40(2.85)$ & $24.41(3.25)$ & $24.33(3.30)$ & $24.69(3.14)$ & $0.009 *$ \\
\hline Atopy, yes, n (\%) & $63(77.78)$ & $34(80.95)$ & $29(74.36)$ & $74(63.79)$ & $58(63.74)$ & $16(64.00)$ & $0.036 *$ \\
\hline \multicolumn{8}{|l|}{ Smoking history } \\
\hline Yes, no quit, n (\%) & $18(22.22)$ & $10(23.81)$ & $8(20.51)$ & $14(12.07)$ & $12(13.19)$ & $2(8)$ & $0.002^{*}$ \\
\hline Yes, quit, n (\%) & $11(13.58)$ & $5(11.90)$ & $6(15.39)$ & $4(3.45)$ & $2(2.19)$ & $2(8)$ & \\
\hline No, n (\%) & $52(64.20)$ & $27(64.29)$ & $25(64.10)$ & $98(84.48)$ & $77(84.62)$ & $21(84)$ & \\
\hline $\begin{array}{l}\text { Pack years of cigarette smoking (pack-years), } \\
\text { mean (SD) }\end{array}$ & $6.66(4.38)$ & $6.47(4.41)$ & $6.86(4.50)$ & $5.17(2.31)$ & $5.00(2.35)$ & $5.75(2.36)$ & 0.191 \\
\hline Length of hospital stay, (days), mean (SD) & $6.75(2.76)$ & $6.29(2.63)$ & $7.26(2.84)$ & $7.09(3.50)$ & $6.79(2.8 \mathrm{I})$ & $8.16(5.23)$ & 0.475 \\
\hline Asthma duration, (months), median (QI, Q3) & $\begin{array}{l}60(12.00 \\
240.00)\end{array}$ & $\begin{array}{l}42(11.75 \\
240.00)\end{array}$ & $\begin{array}{l}96(24.00 \\
240.00)\end{array}$ & $36(5.00,96.00)$ & $24(3.00,84.00)$ & $\begin{array}{l}72(24.00 \\
120.00)\end{array}$ & $0.002 *$ \\
\hline Family history of asthma, $\mathrm{n}(\%)$ & $21(25.93)$ & $12(28.57)$ & $9(23.08)$ & $7(6.03)$ & $6(6.59)$ & I (4.00) & $0.000^{*}$ \\
\hline ACT score, Median (QI, Q3) & $\begin{array}{l}19.00(18.00 \\
20.00)\end{array}$ & $\begin{array}{l}19.5(19.00 \\
21.00)\end{array}$ & $\begin{array}{l}18.00(17.00 \\
18.00)\end{array}$ & $\begin{array}{l}20.00(19.00 \\
21.00)\end{array}$ & $\begin{array}{l}20.00(19.00 \\
21.00)\end{array}$ & $\begin{array}{l}19.00(17.00 \\
19.50)\end{array}$ & $0.000 *$ \\
\hline FeNO, (ppb), median (QI, Q3) & $\begin{array}{l}35.00(16.00 \\
64.25)\end{array}$ & $\begin{array}{l}29.50(17.00 \\
59.25)\end{array}$ & $\begin{array}{l}38.50(15.25 \\
82.25)\end{array}$ & $\begin{array}{l}20.5(12.00, \\
43.50)\end{array}$ & $\begin{array}{l}22.00(12.00 \\
45.00)\end{array}$ & $\begin{array}{l}17.00(12.00 \\
32.00)\end{array}$ & $0.006 *$ \\
\hline \multicolumn{8}{|l|}{ Lung function } \\
\hline $\mathrm{FEV}_{1},(\mathrm{~L})$, mean (SD) & $1.85(0.74)$ & $1.99(0.68)$ & $1.70(0.78)$ & $2.60(0.74)$ & $2.66(0.75)$ & $2.35(0.65)$ & $0.000 *$ \\
\hline $\mathrm{FEV}_{1} \%$ pred, $(\%)$, mean $(\mathrm{SD})$ & $63.54(17.78)$ & $65.36(17.31)$ & $61.57(18.27)$ & $95.04(14.59)$ & $96.24(14.94)$ & $90.66(12.54)$ & $0.000 *$ \\
\hline FVC, (L), mean (SD) & $3.08(1.00)$ & $3.34(0.94)$ & $2.81(1.01)$ & $3.38(0.90)$ & $3.44(0.91)$ & $3.19(0.88)$ & $0.029 *$ \\
\hline FVC\%pred, (\%), mean (SD) & $87.72(17.48)$ & $90.60(18.33)$ & $84.62(16.17)$ & $103.75(15.87)$ & $104.03(16.70)$ & $102.70(12.62)$ & $0.000^{*}$ \\
\hline $\mathrm{FEV}_{1} / \mathrm{FVC}$, (\%), mean (SD) & $58.41(8.54)$ & $58.23(9.25)$ & $58.60(0.78)$ & $76.45(4.52)$ & $76.92(4.61)$ & $74.74(3.81)$ & $0.000^{*}$ \\
\hline \multicolumn{8}{|l|}{ Corticosteroids use for wheezing } \\
\hline $\begin{array}{l}\text { Systemic corticosteroids, (mg), median (QI, } \\
\text { Q3) }\end{array}$ & $0(0,0)$ & $0(0,0)$ & $0(0,40)$ & $0(0,0)$ & $0(0,0)$ & $0(0,40)$ & 0.940 \\
\hline Inhaled budesonide, (mg), mean (SD) & $2.90(0.98)$ & $2.59(1.03)$ & $3.23(0.81)$ & $2.64(1.24)$ & $2.35(1.16)$ & $3.36(1.15)$ & 0.141 \\
\hline
\end{tabular}

Note: *Represents $p<0.05$; suggests statistical significance.

Abbreviations: SD, standard deviation; BMI, body mass index; ACT, asthma control test; QI, quartile I; Q3, quartile 3; FEV 1 , forced expiratory volume in I s; FEV $\%$ pred, FEVI percentage of predicted; FVC, forced vital capacity; FVC\%pred, FVC percentage of predicted.

95\% CI: $1.044-1.216 ; p=0.002)$, and two days before asthma onset (Lag2) $(\mathrm{OR}=1.099 ; 95 \% \mathrm{CI}: 1.019-1.185$; $p=0.014$ ) was also the independent risk factor for hospital admissions for asthma patients with RO, while other air pollutants, including $\mathrm{SO} 2, \mathrm{NO} 2, \mathrm{O} 3$ and $\mathrm{CO}$, could not be proven significant in multivariate analysis. However, no significant association was found between major air pollutant exposure and hospital admissions for asthma patients with FO (Tables 3 and 4).

\section{ROC Curves to Predict Severe Exacerbation in Asthma Patients with RO} We performed an ROC curve analysis for identifying the effects of major air pollutants on the severity of asthma in patients with RO. PM2.5 on Lag0 (AUC = 0.645; $p=0.027$ ) provided the best performance to predict severe asthma exacerbations with RO, with a sensitivity of $36.0 \%$ and a specificity of $91.2 \%$. The AUC of PM10 on Lag0 percentage was $0.641(p=0.031)$, and its optimal cut-off 
Table 2 Comparison of Air Particle Levels Within Fixed Airflow Obstruction Group and Reversible Airflow Obstruction Group

\begin{tabular}{|c|c|c|c|c|c|c|}
\hline & \multicolumn{3}{|c|}{ Fixed Airflow Obstruction Group } & \multicolumn{3}{|c|}{ Reversible Airflow Obstruction Group } \\
\hline & Mild-to-Moderate & Severe & $p$-value & Mild-to-Moderate & Severe & $p$-value \\
\hline \multicolumn{7}{|l|}{ Air particles } \\
\hline \multicolumn{7}{|l|}{ PMI0 (ug/m³), Median (QI, Q3) } \\
\hline Lag0 & $18.14(11.38,33.12)$ & $24.81(18.27,42.58)$ & 0.094 & $20.7(12.65,38.35)$ & $32.76(16.64,63.84)$ & $0.031 *$ \\
\hline Lagl & $19.02(12.87,28.12)$ & $29.22(13.64,41.64)$ & 0.178 & $19.47(12.98,34.83)$ & $34.89(17.34,49.43)$ & $0.032^{*}$ \\
\hline Lag2 & $19.26(12.17,27.47)$ & $26.06(11.86,42.49)$ & 0.342 & $19.47(13.40,34.89)$ & $32.36(16.28,46.57)$ & 0.069 \\
\hline Lag3 & $17.81(12.60,33.54)$ & $26.78(13.02,37.22)$ & 0.502 & $19.67(11.33,38.47)$ & $32.72(19.31,52.46)$ & $0.008^{*}$ \\
\hline \multicolumn{7}{|l|}{ PM2.5 (ug/m³), Median (QI, Q3) } \\
\hline Lag0 & $14.11(8.86,23.12)$ & $19.80(14.82,24.60)$ & 0.133 & $16.08(9.51,25.02)$ & $23.15(12.27,44.18)$ & $0.027^{*}$ \\
\hline Lagl & $14.44(10.42,20.96)$ & $20.48(10.38,25.95)$ & 0.166 & $16.24(10.02,22.71)$ & $24.96(13.09,31.20)$ & $0.033^{*}$ \\
\hline Lag2 & $15.20(10.49,21.80)$ & $20.61(9.59,28.54)$ & 0.275 & $15.69(9.65,26.29)$ & $22.42(12.69,29.30)$ & 0.115 \\
\hline Lag3 & $14.34(9.63,23.54)$ & $20.54(10.59,22.01)$ & 0.473 & $15.69(8.66,26.56)$ & $22.71(14.41,34.77)$ & $0.023 *$ \\
\hline \multicolumn{7}{|l|}{ PMI.0 (ug/m³), Median (QI, Q3) } \\
\hline Lag0 & $11.42(7.06,19.03)$ & $15.17(12.07,21.20)$ & 0.148 & $|2.7|(7.72,21.02)$ & $18.09(8.72,38.76)$ & $0.043^{*}$ \\
\hline Lagl & $11.52(7.21,17.91)$ & $16.42(7.96,22.68)$ & 0.147 & $12.82(7.14,18.11)$ & $21.62(8.86,27.32)$ & 0.071 \\
\hline Lag2 & $10.55(7.52,17.45)$ & $16.70(7.67,23.47)$ & 0.184 & $12.88(7.66,22.27)$ & $18.89(8.64,26.11)$ & 0.243 \\
\hline Lag3 & $11.27(7.03,19.48)$ & $15.86(8.33,19.32)$ & 0.326 & $|2.8|(7.00,22.57)$ & $19.96(8.92,29.28)$ & 0.087 \\
\hline \multicolumn{7}{|l|}{$\mathrm{SO}_{2}\left(\mathrm{ug} / \mathrm{m}^{3}\right)$, Median (QI, Q3) } \\
\hline Lag0 & $3.09(2.22,4.08)$ & $3.08(2.05,4.64)$ & 0.860 & $3.13(2.31,4.26)$ & $3.61(2.68,4.89)$ & 0.289 \\
\hline Lagl & $3.13(2.41,4.16)$ & $3.00(2.37,4.11)$ & 0.941 & $3.11(2.28,4.13)$ & $3.14(2.25,4.76)$ & 0.562 \\
\hline Lag2 & $3.21(2.26,4.16)$ & $3.07(2.14,4.53)$ & 0.857 & $3.20(2.27,4.47)$ & $3.07(2.33,4.86)$ & 0.727 \\
\hline Lag3 & $3.29(2.39,4.10)$ & $3.16(2.06,4.38)$ & 0.859 & $2.96(2.18,4.50)$ & $3.55(2.75,4.61)$ & 0.170 \\
\hline \multicolumn{7}{|l|}{$\mathrm{NO}_{2}\left(\mathrm{ug} / \mathrm{m}^{3}\right)$, Median $(\mathrm{Q}, \mathrm{Q} 3)$} \\
\hline Lag0 & $8.74(5.70,13.33)$ & $11.05(5.92,15.38)$ & 0.427 & $10.03(5.27,13.61)$ & |3.55 (7.5|, 17.33) & 0.061 \\
\hline Lagl & $9.04(5.67,14.26)$ & $10.62(5.78,14.26)$ & 0.864 & $9.33(6.19,14.49)$ & $13.13(6.01,17.27)$ & 0.166 \\
\hline Lag2 & $8.23(6.04,12.70)$ & $8.53(6.02,13.33)$ & 0.918 & $9.64(5.80,14.21)$ & $12.45(7.04,15.90)$ & 0.163 \\
\hline Lag3 & $10.19(6.86,15.22)$ & $11.17(6.36,13.20)$ & 0.473 & $7.97(4.92,12.63)$ & $14.48(5.64,19.17)$ & $0.019 *$ \\
\hline \multicolumn{7}{|l|}{$\mathrm{O}_{3}\left(\mathrm{ug} / \mathrm{m}^{3}\right)$, Median (QI, Q3) } \\
\hline Lag0 & $28.91(16.56,46.86)$ & $27.72(18.45,45.94)$ & 0.739 & $30.20(22.61,39.92)$ & $28.83(21.34,40.57)$ & 0.976 \\
\hline Lagl & $28.74(17.34,44.68)$ & $28.10(18.56,42.53)$ & 0.895 & $29.26(20.64,40.14)$ & $24.90(18.40,39.64)$ & 0.273 \\
\hline Lag2 & $26.34(16.87,46.86)$ & $33.79(15.95,43.82)$ & 0.728 & $29.60(20.21,38.94)$ & $31.99(20.19,44.32)$ & 0.687 \\
\hline Lag3 & $22.98(17.11,36.74)$ & $25.23(13.28,42.83)$ & 0.836 & $31.73(21.18,42.70)$ & $28.08(14.45,39.49)$ & 0.178 \\
\hline \multicolumn{7}{|l|}{$\mathrm{CO}\left(\mathrm{mg} / \mathrm{m}^{3}\right)$, Median (QI, Q3) } \\
\hline Lag0 & $0.46(0.39,0.61)$ & $0.49(0.35,0.58)$ & 0.992 & $0.47(0.38,0.59)$ & $0.48(0.36,0.74)$ & 0.341 \\
\hline Lagl & $0.47(0.35,0.58)$ & $0.45(0.38,0.60)$ & 0.973 & $0.48(0.40,0.58)$ & $0.49(0.34,0.71)$ & 0.321 \\
\hline Lag2 & $0.49(0.39,0.60)$ & $0.46(0.37,0.58)$ & 0.593 & $0.47(0.38,0.57)$ & $0.44(0.34,0.64)$ & 0.732 \\
\hline Lag3 & $0.45(0.39,0.58)$ & $0.49(0.35,0.61)$ & 0.799 & $0.46(0.37,0.56)$ & $0.47(0.34,0.70)$ & 0.760 \\
\hline \multicolumn{7}{|l|}{ Weather } \\
\hline \multicolumn{7}{|l|}{ Temperature $\left({ }^{\circ} \mathrm{C}\right)$, Median $(\mathrm{Q}$ I, Q3) } \\
\hline Lag0 & $26.09(22.03,28.86)$ & $22.88(18.00,26.89)$ & $0.049 *$ & $24.69(21.10,29.09)$ & $23.50(18.01,28.63)$ & 0.317 \\
\hline Lagl & $25.62(22.51,28.79)$ & $22.89(17.42,26.82)$ & 0.056 & $25.15(20.81,28.88)$ & $21.95(17.38,27.39)$ & 0.157 \\
\hline Lag2 & $24.95(22.98,29.06)$ & $22.59(17.53,26.92)$ & 0.057 & $25.62(20.66,28.58)$ & $21.87(18.15,27.14)$ & 0.188 \\
\hline Lag3 & $25.40(23.15,28.94)$ & $22.03(16.81,27.84)$ & $0.025 *$ & $25.23(20.90,28.67)$ & $21.00(16.81,27.98)$ & 0.086 \\
\hline \multicolumn{7}{|l|}{ Humidity (\%), Median (QI, Q3) } \\
\hline Lag0 & $79.38(75.70,82.31)$ & $76.25(67.21,82.75)$ & 0.247 & $77.00(70.79,84.33)$ & $75.46(59.52,80.63)$ & 0.103 \\
\hline Lagl & $80.17(73.40,83.60)$ & $76.83(65.83,80.88)$ & 0.098 & $77.79(77.21,84.58)$ & $75.35(67.65,81.55)$ & 0.170 \\
\hline Lag2 & $78.48(74.77,82.45)$ & $74.29(68.67,81.04)$ & 0.333 & $79.96(72.08,84.17)$ & $75.00(68.31,84.34)$ & 0.268 \\
\hline Lag3 & $79.85(72.45,84.06)$ & $77.21(68.96,84.63)$ & 0,985 & $79.42(70.79,84.54)$ & $74.38(68.90,82.17)$ & 0.227 \\
\hline
\end{tabular}

Note: *Represents $p<0.05$; suggests statistical significance.

Abbreviations: PM, particulate matter; $\mathrm{SO}_{2}$, sulfur dioxide; $\mathrm{NO}_{2}$, nitrogen dioxide; $\mathrm{O}_{3}$, ozone, $\mathrm{CO}$, carbon monoxide; QI, quartile I; $\mathrm{Q} 3$, quartile 3; Lag0, the day of asthma onset; LagI, one day before asthma onset; Lag2, two days before asthma onset; Lag3, three days before asthma onset. 
Table 3 Univariate Logistics Regression Analysis for Fixed Airflow Obstruction Group and Reversible Airflow Obstruction Group

\begin{tabular}{|c|c|c|c|c|c|c|}
\hline & \multicolumn{3}{|c|}{ Fixed Airflow Obstruction Group } & \multicolumn{3}{|c|}{ Reversible Airflow Obstruction Group } \\
\hline & OR & $95 \% \mathrm{Cl}$ & $p$-value & OR & $95 \% \mathrm{Cl}$ & $p$-value \\
\hline \multicolumn{7}{|c|}{ Air particle } \\
\hline \multicolumn{7}{|c|}{ PMIO (ug/m³) } \\
\hline Lag0 & 1.025 & $0.995 \sim 1.057$ & 0.108 & 1.032 & $1.009 \sim 1.055$ & $0.006 *$ \\
\hline LagI & 1.019 & $0.991 \sim 1.048$ & 0.180 & 1.027 & $1.002 \sim 1.052$ & $0.031 *$ \\
\hline Lag2 & 1.009 & $0.984 \sim 1.036$ & 0.476 & 1.028 & $1.001 \sim 1.055$ & $0.038 *$ \\
\hline Lag3 & 1.006 & $0.981 \sim 1.033$ & 0.625 & 1.037 & $1.012 \sim 1.063$ & $0.004 *$ \\
\hline \multicolumn{7}{|c|}{ PM2.5 (ug/m³) } \\
\hline Lag0 & 1.027 & $0.980 \sim 1.076$ & 0.273 & 1.051 & $1.016 \sim 1.087$ & $0.004 *$ \\
\hline LagI & 1.023 & $0.982 \sim 1.065$ & 0.273 & 1.036 & $0.999 \sim 1.073$ & 0.054 \\
\hline Lag2 & 1.013 & $0.975 \sim 1.052$ & 0.513 & 1.030 & $0.993 \sim 1.069$ & 0.108 \\
\hline Lag3 & 1.005 & $0.969 \sim 1.043$ & 0.781 & 1.044 & $1.007 \sim 1.081$ & $0.018^{*}$ \\
\hline \multicolumn{7}{|c|}{ PMI.0 (ug/m³) } \\
\hline Lag0 & 1.028 & $0.978 \sim 1.080$ & 0.273 & 1.053 & $1.016 \sim 1.092$ & $0.005 *$ \\
\hline LagI & 1.026 & $0.982 \sim 1.071$ & 0.250 & 1.036 & $0.996 \sim 1.076$ & 0.076 \\
\hline Lag2 & 1.016 & $0.976 \sim 1.059$ & 0.435 & 1.026 & $0.987 \sim 1.067$ & 0.186 \\
\hline Lag3 & 1.008 & $0.970 \sim 1.048$ & 0.678 & 1.039 & $1.000 \sim 1.078$ & $0.047^{*}$ \\
\hline \multicolumn{7}{|c|}{$\mathrm{SO}_{2}\left(\mathrm{ug} / \mathrm{m}^{3}\right)$} \\
\hline Lag0 & 1.029 & $0.747 \sim 1.417$ & 0.863 & 1.184 & $0.898 \sim 1.561$ & 0.232 \\
\hline LagI & 0.966 & $0.717 \sim 1.301$ & 0.820 & 1.105 & $0.855 \sim 1.429$ & 0.445 \\
\hline Lag2 & 0.933 & $0.705 \sim 1.233$ & 0.625 & 1.102 & $0.839 \sim 1.446$ & 0.485 \\
\hline Lag3 & 0.958 & $0.702 \sim 1.309$ & 0.789 & 1.150 & $0.891 \sim 1.484$ & 0.283 \\
\hline \multicolumn{7}{|c|}{$\mathrm{NO}_{2}\left(\mathrm{ug} / \mathrm{m}^{3}\right)$} \\
\hline Lag0 & 1.013 & $0.941 \sim 1.092$ & 0.725 & 1.049 & $0.997 \sim 1.104$ & 0.064 \\
\hline LagI & 1.011 & $0.942 \sim 1.085$ & 0.761 & 1.016 & $0.964 \sim 1.071$ & 0.558 \\
\hline Lag2 & 0.977 & $0.914 \sim 1.044$ & 0.489 & 1.039 & $0.973 \sim 1.109$ & 0.257 \\
\hline Lag3 & 0.955 & $0.882 \sim 1.034$ & 0.259 & 1.091 & $1.025 \sim 1.161$ & $0.006 *$ \\
\hline \multicolumn{7}{|c|}{$\mathrm{O}_{3}\left(\mathrm{ug} / \mathrm{m}^{3}\right)$} \\
\hline Lag0 & 0.996 & $0.974 \sim 1.018$ & 0.712 & 1.000 & $0.97 \mid \sim 1.030$ & 0.998 \\
\hline LagI & 0.996 & $0.974 \sim 1.019$ & 0.738 & 0.986 & $0.956 \sim 1.018$ & 0.392 \\
\hline Lag2 & 1.003 & $0.980 \sim 1.027$ & 0.799 & 1.002 & $0.973 \sim 1.032$ & 0.883 \\
\hline Lag3 & 1.003 & $0.977 \sim 1.029$ & 0.846 & 0.978 & $0.949 \sim 1.008$ & 0.153 \\
\hline \multicolumn{7}{|c|}{$\mathrm{CO}\left(\mathrm{mg} / \mathrm{m}^{3}\right)$} \\
\hline Lag0 & 0.522 & $0.035 \sim 7.907$ & 0.639 & 4.425 & $0.429 \sim 45.636$ & 0.212 \\
\hline LagI & 0.846 & $0.064 \sim 11.190$ & 0.889 & 6.460 & $0.443 \sim 94.178$ & 0.172 \\
\hline Lag2 & 0.389 & $0.022 \sim 6.814$ & 0.518 & 0.881 & $0.066 \sim 11.754$ & 0.923 \\
\hline Lag3 & 0.622 & $0.055 \sim 7.047$ & 0.702 & 0.879 & $0.368 \sim 2.098$ & 0.772 \\
\hline \multicolumn{7}{|c|}{ Weather } \\
\hline \multicolumn{7}{|c|}{ Temperature $\left({ }^{\circ} \mathrm{C}\right)$} \\
\hline Lag0 & 0.919 & $0.841 \sim 1.005$ & 0.064 & 0.947 & $0.867 \sim 1.033$ & 0.218 \\
\hline LagI & 0.899 & $0.817 \sim 0.988$ & $0.028^{*}$ & 0.937 & $0.858 \sim 1.022$ & 0.143 \\
\hline Lag2 & 0.895 & $0.816 \sim 0.982$ & $0.019 *$ & $0.94 I$ & $0.863 \sim 1.026$ & 0.168 \\
\hline $\operatorname{Lag} 3$ & 0.890 & $0.814 \sim 0.973$ & $0.011^{*}$ & 0.919 & $0.845 \sim 0.999$ & $0.049 *$ \\
\hline \multicolumn{7}{|c|}{ Humidity (\%) } \\
\hline Lag0 & 0.978 & $0.943 \sim 1.015$ & 0.978 & 0.971 & $0.937 \sim 1.006$ & 0.106 \\
\hline LagI & 0.963 & $0.921 \sim 1.008$ & 0.102 & 0.975 & $0.941 \sim 1.011$ & 0.173 \\
\hline Lag2 & 0.981 & $0.943 \sim 1.020$ & 0.331 & 0.981 & $0.949 \sim 1.015$ & 0.269 \\
\hline Lag3 & 1.000 & $0.964 \sim 1.037$ & 0.985 & 0.981 & $0.951 \sim 1.012$ & 0.229 \\
\hline
\end{tabular}

Note: *Represents $p<0.05$; suggests statistical significance.

Abbreviations: PM, particulate matter; $\mathrm{SO}_{2}$, sulfur dioxide; $\mathrm{NO}_{2}$, nitrogen dioxide; $\mathrm{O}_{3}$, ozone, $\mathrm{CO}$, carbon monoxide; Lag0, the day of asthma onset; LagI, one day before asthma onset; Lag2, two days before asthma onset; Lag3, three days before asthma onset; OR, odds ratio; $\mathrm{Cl}$, confidence interval. 
Table 4 Multivariable Logistics Regression Analysis for Reversible Airflow Obstruction Group

\begin{tabular}{|c|c|c|c|c|c|c|c|c|c|}
\hline & \multicolumn{3}{|c|}{ PMIO } & \multicolumn{3}{|c|}{ PM2.5 } & \multicolumn{3}{|c|}{ PMI } \\
\hline & OR & $95 \% \mathrm{Cl}$ & $p$ & OR & $95 \% \mathrm{Cl}$ & $P$ & OR & $95 \% \mathrm{Cl}$ & $p$ \\
\hline \multicolumn{10}{|l|}{ PMIO (ug/m³) } \\
\hline Lag0 & $\mathrm{I} .057$ & $1.002 \sim 1.115$ & $0.042^{*}$ & I & I & l & l & I & l \\
\hline Lag I & 1.127 & $1.044 \sim 1.216$ & $0.002^{*}$ & 1 & I & l & I & l & l \\
\hline Lag2 & 1.099 & $1.019 \sim 1.185$ & $0.014^{*}$ & 1 & I & 1 & 1 & 1 & 1 \\
\hline Lag3 & 1.058 & $0.988 \sim 1.133$ & 0.106 & I & I & I & I & I & I \\
\hline \multicolumn{10}{|l|}{ PM2.5 (ug/m $\left.\mathrm{m}^{3}\right)$} \\
\hline Lag0 & I & I & I & 1.130 & $1.033 \sim 1.236$ & $0.007^{*}$ & I & I & I \\
\hline Lag3 & I & I & l & 1.038 & $0.951 \sim 1.132$ & 0.401 & I & I & l \\
\hline \multicolumn{10}{|l|}{ PMI.0 (ug/m³) } \\
\hline Lag0 & I & I & I & l & I & I & 1.150 & $1.035 \sim 1.277$ & $0.009 *$ \\
\hline Lag3 & I & I & I & 1 & I & I & 1.004 & $0.912 \sim 1.105$ & 0.936 \\
\hline \multicolumn{10}{|l|}{$\mathrm{NO}_{2}\left(\mathrm{ug} / \mathrm{m}^{3}\right)$} \\
\hline Lag3 & 1.023 & $0.881 \sim 1.188$ & 0.765 & 1.079 & $0.943 \sim 1.235$ & 0.269 & 1.120 & $0.981 \sim 1.279$ & 0.095 \\
\hline \multicolumn{10}{|l|}{ Temperature $\left({ }^{\circ} \mathrm{C}\right)$} \\
\hline Lag3 & 0.487 & $0.911 \sim 1.216$ & 0.487 & 1.033 & $0.897 \sim 1.190$ & 0.652 & 1.009 & $0.875 \sim 1.163$ & 0.900 \\
\hline
\end{tabular}

Note: *Represents $p<0.05$; suggest statistical significance.

Abbreviations: $\mathrm{PM}$, particulate matter; $\mathrm{SO}_{2}$, sulfur dioxide; $\mathrm{NO}_{2}$, nitrogen dioxide; $\mathrm{O}_{3}$, ozone, $\mathrm{CO}$, carbon monoxide; Lag0, the day of asthma onset; LagI, one day before asthma onset; Lag2, two days before asthma onset; Lag3, three days before asthma onset; OR, odds ratio; Cl, confidence interval.

value was $57.659 \mu \mathrm{g} / \mathrm{m}^{3}$, with $28.0 \%$ sensitivity and 98.9\% specificity, and the AUC of PM1.0 on Lag0 percentage was $0.633(p=0.043)$, and its optimal cut-off value was $29.271 \mu \mathrm{g} / \mathrm{m}^{3}$, with $36.0 \%$ sensitivity and $92.3 \%$ specificity. Moreover, at a cut-off value of $26.419 \mu \mathrm{g} / \mathrm{m}^{3}$, the sensitivity and specificity of PM10 on Lag1 (AUC $=0.640 ; p=0.032$ ) were $68.0 \%$ and $68.1 \%$, respectively. The diagnostic performance of major air pollutant exposure for severe asthma exacerbations with $\mathrm{RO}$ is shown in Table 5 and Figure 3.

\section{Discussion}

In the study, we observed that short-term periods of PM10, PM2.5 and PM1.0 elevation were matched with the timing increases in severe asthma exacerbations with RO. Our study also demonstrated that PM10 on Lag0, PM2.5 on Lag0, PM1 on Lag0, PM10 on Lag1 and Lag2 were the health risk factors of severe asthma patients with RO, which suggested that short-term exposure to higher concentrations of PM 10, PM2.5 and PM1 is associated with exacerbation severity of asthma patients with RO.

Our study assessed 197 adult patients who had acute exacerbation of asthma, and $58.9 \%$ of them presented with asthma with RO. We observed that the asthma group with RO had shorter disease duration, a lower percentage of men, a lower rate of smoking and family history and lower FeNO levels when compared with the group with FO. Since smoking habits might be more risk-inducing in men, it was suggested that the asthma with FO was linked with smoking-induced airway damage, longer courses of

Table 5 ROC Curves to Predict Severe Exacerbations in Asthma Patients with Reversible Airflow Obstruction

\begin{tabular}{|l|c|c|c|c|c|c|}
\hline & AUC & $\mathbf{9 5 \%}$ Cl & Cut-Off Value & Sensitivity & Specificity & P \\
\hline PMI0 (Lag0) & 0.641 & $0.514 \sim 0.769$ & 57.659 & 0.280 & 0.989 & $0.03 I^{*}$ \\
PMI0 (LagI) & 0.640 & $0.511 \sim 0.976$ & 26.419 & 0.680 & 0.681 & $0.032^{*}$ \\
PM2.5 (Lag0) & 0.645 & $0.513 \sim 0.777$ & 32.059 & 0.360 & 0.912 & $0.027^{*}$ \\
PMI.0 (Lag0) & 0.633 & $0.494 \sim 0.772$ & 29.271 & 0.360 & 0.923 & $0.043^{*}$ \\
\hline
\end{tabular}

Note: *Represents $p<0.05$; suggests statistical significance.

Abbreviations: PM, particulate matter; Lag0, the day of asthma onset; Lagl, one day before asthma onset; Lag3, three days before asthma onset; AUC, the area under the $\mathrm{ROC}$ curve; $\mathrm{Cl}$, confidence interval. 


\section{ROC Curve}

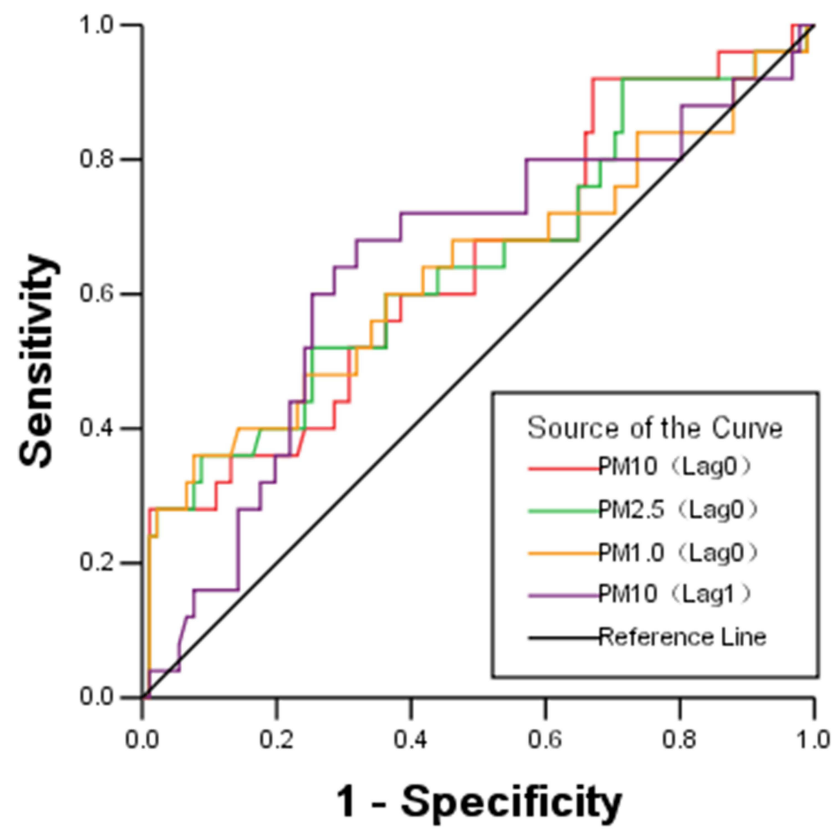

Figure 3 Receiver operating characteristic (ROC) curves for PM exposure on different lag days (Lag03) to affect severity in asthma patients with reversible airway obstruction (RO).

disease and more eosinophilic inflammation. All these factors were implicated as playing an important role in airway remodeling, and ultimately FO in asthma patients. Moreover, we also found that the levels of large airway parameters including FEV1, FEV1\%pred, FVC, FVC\% pred, and FEV1/FVC were significantly decreased in the FO group compared with the RO group. These results showed the declines in ventilatory function among the patients, which are consistent with the prolonged disease duration of asthma patients with FO. Some researchers found there was an increased risk of $\mathrm{FO}$ in child patients. ${ }^{15}$ It may indicate that long-term complications of asthma can lead to airway remodeling, and be responsible for the accelerated loss of lung function.

Previous reports have shown that exposure to ambient air pollution was associated with acute exacerbation of asthma and severe asthma attack, ${ }^{16-18}$ but less is known about the relationship between the exposure levels of particle pollutants and asthma patients with or without FO. In the study, we found that short-term exposure to PM10, PM2.5 and PM1 (on lags of 1-3 days) was associated with severe exacerbations in asthma patients with RO, and may be the independent risk factor for these adverse asthma events. However, no significant difference was found in the exposure levels of air particles (on lags of 1-3 days) between severe and mild-to-moderate asthma patients in the FO group. These data provide new evidence that short-term exposure to PM (10-1) may be closely linked to severe exacerbation in asthma with RO but not FO.

Studies have also shown that exposure to ambient PM collected in urban Baltimore increased the pathophysiological features of asthma via activation of lymphocytedependent pathways. ${ }^{19}$ It is speculated that PM exposure may play a significant role in the increased severity of allergic asthma. Other studies demonstrated that short-term PM exposure might also increase asthma mortality risk. ${ }^{20}$ If these severe exacerbations are coupled with deleterious effects caused by acute PM exposure, they could inform an intervention to reduce hospital admissions and avoid lung function decline for asthma patients with RO. We then analyzed whether acute PM exposure was responsible for increased severity for asthma patients with RO by performing AUC calculations. The results showed that, on Lag0, PM2.5 had the best AUC value, of 0.645 (cut-off value $32.059 \mathrm{ug} / \mathrm{m}^{3}$ ), with a sensitivity of $36.0 \%$ and a specificity of $91.2 \%$. Moreover, when the concentration of PM10 reached the cutoff values (57.659 and $26.419 \mathrm{ug} / \mathrm{m}^{3}$ ) on Lag0 and Lag1, the sensitivity and specificity for Lag0 were $28.0 \%$ and $98.9 \%$ and for Lag1 were $68.0 \%$ and $68.1 \%$, respectively. It was suggested that exposure to higher concentrations of PM (PM1, 2.5 and 10) on different lag days might increase the severity of asthma patients with RO, and have a harmful impact on severe acute exacerbations of asthma. Thus, asthma patients with RO should restrict outdoor activities when ambient PM levels are high, so that they may reduce the chance of having a severe asthma attack. Interestingly, our study did not find a statistically significant association between short-term exposure to PM and increased severe exacerbations in asthma with FO. Further research is essential to find out whether or not PM exposure may affect exacerbation severity of asthma with FO.

There are some limitations in this study. First, it is a single-centre study with a limited number of subjects, which may be a source of potential bias or a confounding factor. Additionally, due to the small number of patients in the RO group, it is difficult for the ROC curve to explicitly show the effect of air pollutant exposure on severe asthma. Second, we did not study the clinical phenotype like earlyonset asthma and late-onset asthma due to the limitations of time, manpower, and cost. Third, we did not explore the mechanism through which ambient air particles cause acute exacerbation of asthma with RO. Finally, several important factors that might affect the association between air pollutant 
exposure and asthma exacerbation may be considered, such as health insurance coverage, housing quality, outdoor activities, holidays and peak flu periods. Nevertheless, the retrospective design of this study limits the validity of relevant data, and recall accuracy is uncertain for up to two years. In the future, we will enlarge the sample size, consider more factors that interfere with the trend in pollutants, and conduct outpatient follow-up visits after hospital discharge.

In conclusion, this study showed that short-term exposure to PM10, PM2.5 and PM1 on different lag days may be the risk factors for acute exacerbations, and may also be associated with worse severity of asthma patients with RO. However, no significant association between PM exposure on lag 0-3 days and acute exacerbations in asthma patients with FO was found. These results suggest that PM exposure in the short term may play a role in frequency of attacks and severity of symptoms among asthma patients with RO, and education and intervention are needed to reduce environmental triggers and enhance respiratory outcomes. Further longitudinal studies are needed to verify and confirm the results.

\section{Statement of Ethics}

This research protocol was approved by the Medical Ethics Committee of Shenzhen People's Hospital, and all subjects have signed written informed consent forms before enrolment in the study.

\section{Acknowledgments}

The study was supported by the Natural Science Foundation of Guangdong Province, China (Project Number: 2020A151501040), the Natural Science Foundation of China (Project Number: 81300012), and the Appropriate Health Technology Promotion Project of Guangdong Province (Project Number: 2020181). Wei Fang, Yu Zhang, Sinian Li, and Aiming Liu are co-authors of this study.

\section{Disclosure}

The authors have no conflicts of interest to declare.

\section{References}

1. Padem N, Saltoun C. Classification of asthma. Allergy Asthma Proc. 2019;40(6):385-388. doi:10.2500/aap.2019.40.4253

2. Rehman A, Amin F, Sadeeqa S. Prevalence of asthma and its management: a review. J Pak Med Assoc. 2018;68(12):1823-1827.
3. Bertolini F, Carriero V, Bullone $\mathrm{M}$, et al. Correlation of matrix-related airway remodeling and bradykinin B1 receptor expression with fixed airflow obstruction in severe asthma. Allergy. 2021;76 (6):1886-1890. doi:10.1111/all.14691

4. Chipps BE, Hirsch I, Trudo F, et al. Benralizumab efficacy for patients with fixed airflow obstruction and severe, uncontrolled eosinophilic asthma. Ann Allergy Asthma Immunol. 2020;124(1):79-86. doi:10.1016/j.anai.2019.10.006

5. Tashkin DP, Moore GE, Trudo F, et al. Assessment of consistency of fixed airflow obstruction status during budesonide/formoterol treatment and its effects on treatment outcomes in patients with asthma. J Allergy Clin Immunol Pract. 2016;4(4):705-712. doi:10.1016/j. jaip.2016.02.014

6. Milne S, Mannino D, Sin DD. Asthma-COPD overlap and chronic airflow obstruction: definitions, management, and unanswered questions. $J$ Allergy Clin Immunol Pract. 2020;8(2):483-495. doi:10.1016/j.jaip.2019.10.044

7. Ferreira DS, Carvalho-Pinto RM, Gregório MG, et al. Airway pathology in severe asthma is related to airflow obstruction but not symptom control. Allergy. 2018;73(3):635-643. doi:10.1111/all.13323

8. Papi A, Brightling C, Pedersen SE, et al. Asthma. Lancet. 2018;391 (10122):783-800. doi:10.1016/S0140-6736(17)33311-1

9. Vardoulakis S, Osborne N. Air pollution and asthma. Arch Dis Child. 2018;103(9):813-814. doi:10.1136/archdischild-2017-314543

10. Cooper DM, Loxham M. Particulate matter and the airway epithelium: the special case of the underground? Eur Respir Rev. 2019;28 (153):190066. doi:10.1183/16000617.0066-2019

11. Asthma G I F. Global Strategy for asthma management and prevention; 2017. Available from: www.ginasthma.org. Accessed September 2, 2021.

12. Tashkin DP, Chipps BE, Trudo F, et al. Fixed airflow obstruction in asthma: a descriptive study of patient profiles and effect on treatment responses. $J$ Asthma. 2014;51(6):603-609. doi:10.3109/ 02770903.2014.895012

13. Smith BM, Zhao N, Olivenstein R, et al. Asthma and fixed airflow obstruction: long-term trajectories suggest distinct endotypes. Clin Exp Allergy. 2021;51(1):39-48. doi:10.1111/cea.13714

14. American Thoracic Society. ATS/ERS recommendations for standardized procedures for the online and offline measurement of exhaled lower respiratory nitric oxide and nasal nitric oxide. $\mathrm{Am}$ $J$ Respir Crit Care Med. 2005;171(8):912-930. doi:10.1164/ rccm.200406-710ST

15. Mcgeachie MJ, Yates KP, Zhou X, et al. Patterns of growth and decline in lung function in persistent childhood asthma. $N$ Engl J Med. 2016;374(19):1842-1852. doi:10.1056/NEJMoa1513737

16. Erzurum SC. New insights in oxidant biology in asthma. Ann Am Thorac Soc. 2016;13 Suppl 1(Suppl1):S35-9.

17. Li Y, Duan J, Yang M, et al. Transcriptomic analyses of human bronchial epithelial cells BEAS-2B exposed to atmospheric fine particulate matter PM (2.5). Toxicol in Vitro. 2017;42:171-181. doi:10.1016/j.tiv.2017.04.014

18. Pini L, Giordani J, Concoreggi C, et al. Effects of short-term exposure to particulate matter on emergency department admission and hospitalization for asthma exacerbations in Brescia district. $J$ Asthma. Epub 2021 Jun 14. doi:10.1080/02770903.2021.1929310

19. Saunders V, Breysse P, Clark J, et al. Particulate matter-induced airway hyperresponsiveness is lymphocyte dependent. Environ Health Perspect. 2010;118(5):640-646. doi:10.1289/ehp.0901461

20. Liu Y, Pan J, Zhang H, et al. Short-term exposure to ambient air pollution and asthma mortality. Am J Respir Crit Care Med. 2019;200(1):24-32. doi:10.1164/rccm.201810-1823OC 


\section{Publish your work in this journal}

The Journal of Asthma and Allergy is an international, peer-reviewed open-access journal publishing original research, reports, editorials and commentaries on the following topics: Asthma; Pulmonary physiology; Asthma related clinical health; Clinical immunology and the immunological basis of disease; Pharmacological interventions and

Submit your manuscript here: https://www.dovepress.com/journal-of-asthma-and-allergy-journal new therapies. The manuscript management system is completely online and includes a very quick and fair peer-review system, which is all easy to use. Visit http://www.dovepress.com/testimonials.php to read real quotes from published authors. 